\title{
EXISTENCE AND MULTIPLE SOLUTIONS FOR NONAUTONOMOUS SECOND ORDER SYSTEMS WITH NONSMOOTH POTENTIALS
}

\author{
YAN NiNG* AND TIANQING AN
}

\begin{abstract}
This paper is concerned with the nonautonomous second order Hamiltonian systems with nondifferetiable potentials. By using the nonsmooth least action principle and the nonsmooth local linking theorem, we obtain some new existence and multiplicity results for the periodic solutions.
\end{abstract}

\section{Introduction and main results}

In this paper we consider the following second order periodic system with a nonsmooth potential

$$
\left\{\begin{array}{l}
\ddot{u}(t) \in \partial F(t, u(t)) \quad \text { a.e. } t \in[0, T] \\
u(0)-u(T)=\dot{u}(0)-\dot{u}(T)=0
\end{array}\right.
$$

where $T>0$, the potential function $F:[0, T] \times \mathbf{R}^{N} \rightarrow \mathbf{R}$ is locally Lipschitz continuous in $x$ and $\partial F(t, x)$ denotes the Clarke subdifferential of $F$ for $x$.

The system (1) has been studied in the past decades and many excellent results appeared, for example, the work of D. Pasca [10]. Systems driven by the vector p-Laplacian or p-Laplacian-like operators were studied by E. H. Papageorgiou and N. S. Papageorgiou [9], S. Aizicovici and N. S. Papageorgiou [1], D. Pasca [11] and the reference therein. We only focus on the semilinear case (i.e., $p=2$ ) in the present paper, and our approach is based on the nonsmooth least action principle by [4] and a nonsmooth local linking theorem by [6]. It should be noted that our results are different from that of those mentioned above even letting $p=2$ in their theorems. Examples are given to show the difference.

2010 Mathematics Subject Classification. 34C25; 35B38; 49J52.

Key words and phrases. Periodic solutions; Second order systems; Generalized subdifferential; Nonsmooth Cerami condition; Locally Lipschitz function; Nonsmooth local linking theorem.

* Corresponding author.

Received November 13, 2014; revised December 19, 2014. 
When $F(t, x)$ is continuously differentiable in $x$, the problem (1) becomes the second order Hamiltonian systems

$$
\left\{\begin{array}{l}
\ddot{u}(t)=\nabla F(t, u(t)) \quad \text { a.e. } t \in[0, T], \\
u(0)-u(T)=\dot{u}(0)-\dot{u}(T)=0 .
\end{array}\right.
$$

There have been a lot of contributions on problem (2), and we can refer to Mawhin-Willem [7], Tang [12], Tang-Wu [13], Aizmahin-An [2] and so on. In these works, the following assumption is necessary:

(A) $F(t, x)$ is measurable in $t$ for every $x \in \mathbf{R}^{N}$ and continuously differentiable in $x$ for a.e. $t \in[0, T]$, and there exist $a \in C\left(\mathbf{R}^{+}, \mathbf{R}^{+}\right), b \in L^{1}\left(0, T ; \mathbf{R}^{+}\right)$ such that

$$
|F(t, x)| \leq a(|x|) b(t), \quad|\nabla F(t, x)| \leq a(|x|) b(t)
$$

for all $x \in \mathbf{R}^{N}$ and a.e. $t \in[0, T]$, where $\mathbf{R}^{+}$is the set of all nonnegative real number.

Throughout this paper, we always suppose that $F=F_{1}+F_{2}$ with $F_{1}, F_{2}$ satisfying the following assumption $\left(A^{\prime}\right)$ :

- $F_{1}, F_{2}$ are integrable in $t$ over $[0, T]$ for each $x \in \mathbf{R}^{N}$;

- $F_{1}$ is strictly differentiable and $F_{2}$ is locally Lipschitz continuous in $x$ for each $t \in[0, T]$.

Let $H_{T}^{1}$ be the usual Sobolev space with norm

$$
\|u\|=\left(\int_{0}^{T}|u(t)|^{2} d t+\int_{0}^{T}|\dot{u}(t)|^{2} d t\right)^{1 / 2} .
$$

The corresponding functional $\varphi: H_{T}^{1} \rightarrow R$ is given by

$$
\varphi(u)=\frac{1}{2} \int_{0}^{T}|\dot{u}(t)|^{2} d t+\int_{0}^{T} F(t, u(t)) d t .
$$

The main results of this paper are as follows:

THeOREM 1.1. Assume that $F=F_{1}+F_{2}$, where $F_{1}, F_{2}$ satisfy assumption $\left(A^{\prime}\right)$ above and the following conditions:

$\left(i_{1}\right)$ There exists $k \in L^{2}(0, T ; \mathbf{R})$ such that for all $x_{1}, x_{2} \in \mathbf{R}^{N}$ and all $t \in[0, T]$

$$
\left|F_{1}\left(t, x_{1}\right)-F_{1}\left(t, x_{2}\right)\right| \leq k(t)\left|x_{1}-x_{2}\right| .
$$

(i $\left.i_{2}\right)$ There exist $f, g \in L^{\infty}\left(0, T ; \mathbf{R}^{+}\right)$and $\alpha \in[0,1)$ such that for all $x \in \mathbf{R}^{N}$ and a.e. $t \in[0, T]$,

$$
\xi \in \partial F_{2}(t, x) \Rightarrow|\xi| \leq f(t)|x|^{\alpha}+g(t) .
$$

(i $\left.i_{3}\right)$ There exists $h \in L^{1}(0, T)$ such that for a.e. $t \in[0, T]$ and all $x \in \mathbf{R}^{N}$

$$
F_{1}(t, x) \geq h(t)
$$


and

$$
|x|^{-2 \alpha} \int_{0}^{T} F_{2}(t, x) d t \rightarrow+\infty \quad \text { as }|x| \rightarrow \infty .
$$

Then problem (1) possesses at least one solution which minimizes the functional $\varphi$ on $H_{T}^{1}$.

Remark 1.1. The function $F_{1}(t, x)$ is globally Lipschitz continuous in $x$ on $H_{T}^{1}$ provided the condition (3) holds. If $F(t, x)$ is measurable in $t$ for every $x \in \mathbf{R}^{N}$ and continuously differentiable in $x$ for a.e. $t \in[0, T]$, the inequality (4) becomes

$$
\left|\nabla F_{2}(t, x)\right| \leq f(t)|x|^{\alpha}+g(t) .
$$

Then our Theorem 1.1 generalizes Theorem 1 in [12].

Remark 1.2. There are functions $F$ satisfying our Theorem 1.1 but not satisfying the results in $[1,2,7,9-13]$. For example, let $F(t, x)=F_{1}(t, x)+F_{2}(t, x)$ with

$$
F_{1}(t, x)=\frac{t^{2}}{2}|\sin x|, \quad F_{2}(t, x)= \begin{cases}-\frac{\theta(t)}{2}|x|^{2}, & |x| \leq 1, \\ \frac{f(t)}{\alpha+1}|x|^{\alpha+1}-\frac{\theta(t)}{2}-\frac{f(t)}{\alpha+1}, & |x|>1\end{cases}
$$

for all $(t, x) \in[0, T] \times \mathbf{R}^{N}$, where $\alpha \in[0,1), \theta, f \in L^{\infty}\left(0, T ; \mathbf{R}^{+}\right)$.

THEOREM 1.2. Assume that $F=F_{1}+F_{2}$ with $\int_{0}^{T} F(t, 0) d t=0$ and $F_{1}, F_{2}$ satisfy assumptions of Theorem 1.1. Suppose that there exist $\delta>0$ and an integer $k \geq 0$ such that

$$
-\frac{1}{2}(k+1)^{2} \omega^{2}|x|^{2} \leq F(t, x)-F(t, 0) \leq-\frac{1}{2} k^{2} \omega^{2}|x|^{2}
$$

for all $|x| \leq \delta$ and a.e. $t \in[0, T]$, where $\omega=\frac{2 \pi}{T}$. Then problem (1) has at least three distinct solutions in $H_{T}^{1}$.

Remark 1.3. Theorem 1.2 generalizes Theorem 2 in [13] and Thorem 4 in [12]. Suppose that $F=F_{1}+F_{2}$ and

$$
F_{1}(t, x)=\frac{t^{2}}{2}|x|, \quad F_{2}(t, x)= \begin{cases}-\frac{1}{2} \omega^{2}|x|^{2}-\frac{t^{2}}{2}|x|, & |x| \leq 1 \\ \frac{t^{2}}{\alpha+1}|x|^{\alpha+1}-\frac{1}{2} \omega^{2}-\frac{t^{2}}{2}-\frac{t^{2}}{\alpha+1}, & |x|>1\end{cases}
$$

for all $(t, x) \in[0, T] \times \mathbf{R}^{N}$, where $\alpha \in[0,1), \omega=\frac{2 \pi}{T}$. Then the function $F$ satisfies Theorem 1.2 but not satisfies Theorem 2 in [13] and Thorem 4 in [12]. 
THEOREM 1.3. Assume that $F=F_{1}+F_{2}$ with $\int_{0}^{T} F(t, 0) d t=0$ and $F_{1}, F_{2}$ satisfy assumptions of Theorem 1.1. Suppose that

$$
\liminf _{x \rightarrow 0} \frac{F(t, x)}{|x|^{2}} \geq-\frac{2 \pi^{2}}{T^{2}}
$$

for a.e. $t \in[0, T]$, and there exist $\hat{\delta}>0$ such that for all $|x| \leq \hat{\delta}$

$$
\int_{0}^{T} F(t, x) d t \leq 0 .
$$

Then problem (1) has at least three distinct solutions in $H_{T}^{1}$.

Remark 1.4. Theorem 1.3 is new even in the case that $F \in C^{1}$ for system (2). There are functions $F$ satisfying Theorem 1.3 but not satisfying the results in $[1,2,7,9-13]$. For example, let $F=F_{1}+F_{2}$ and

$$
F_{1}(t, x) \equiv 0, \quad F_{2}(t, x)= \begin{cases}-\frac{2 \pi^{2}}{T^{2}}|x|^{2}, & |x| \leq 1, \\ \frac{2 t^{3}}{\alpha+1}|x|^{\alpha+1}-\frac{2 \pi^{2}}{T^{2}}-\frac{2 t^{3}}{\alpha+1}, & |x|>1\end{cases}
$$

for all $(t, x) \in[0, T] \times \mathbf{R}^{N}$, where $\alpha \in[0,1)$.

\section{Basic definitions and preliminary results}

Let $(X,\|\cdot\|)$ be a real Banach space. We denote by $X^{*}$ the dual space of $X$, while $\langle\cdot, \cdot\rangle$ stands for the duality pairing between $X$ and $X^{*}$. A functional $h: X \rightarrow \mathbf{R}$ is called locally Lipschitz continuous if for every $u \in X$ there exist a neighborhood $V_{u}$ of $u$ and a constant $L_{u} \geq 0$ such that

$$
|h(z)-h(w)| \leq L_{u}\|z-w\|, \quad \forall z, w \in V_{u} .
$$

If $u, v \in X$, we write $h^{o}(u ; v)$ for the generalized directional derivative of $h$ at the point $u$ along the direction $v$, i.e.,

$$
h^{\circ}(u ; v):=\limsup _{w \rightarrow u, t \rightarrow 0^{+}} \frac{h(w+t v)-h(w)}{t} .
$$

It is well known that $h^{\circ}$ is upper semicontinuous on $X \times X$ [5, Proposition 2.1.1].

For locally Lipschitz continuous functionals $h_{1}, h_{2}: X \rightarrow \mathbf{R}$, we have

$$
\left(h_{1}+h_{2}\right)^{\circ}(x ; z) \leq h_{1}^{\circ}(x ; z)+h_{2}^{\circ}(x ; z), \quad \forall x, z \in X .
$$

The generalized gradient of the function $h$ in $u$, denoted by $\partial h(u)$, is the set defined by

$$
\partial h(u):=\left\{u^{*} \in X^{*}:\left\langle u^{*}, v\right\rangle \leq h^{\circ}(u ; v), \forall v \in X\right\} .
$$


Proposition 2.1.2 of [5] ensures that $\partial h(u)$ turns out nonempty, convex, weak* compact, thus the function $\lambda(x)=\min _{w \in \partial h(x)}\|w\|_{X^{*}}$ exists and is lower semicontinuous.

We call $h: X \rightarrow R$ is strictly differentiable in $u$ if there exist an element $\xi \in X^{*}$ such that for each $v$,

$$
\lim _{w \rightarrow u, t \rightarrow 0^{+}} \frac{h(w+t v)-h(w)}{t}=\langle\xi, v\rangle,
$$

and provided the convergence is uniform for $v$ in compact sets (This last condition is automatic if $h$ is Lipschitz near $u$, see [5], P30).

If $f, g: X \rightarrow X$ be locally Lipschitz continuous, then

$$
\partial(f+g)(x) \subset \partial f(x)+\partial g(x)
$$

for all $x \in X$. Further, if at least one of the functional $f, g$ is strictly differentiable at $x$ then equality holds, and $\partial f(x)=\left\{f^{\prime}(x)\right\}$ when $f \in C^{1}(X)$.

A point $u \in X$ is said to be a critical point of $h$ if

$$
h^{\circ}(u ; v) \geq 0, \quad \forall v \in X,
$$

which clearly means $\theta \in \partial h(u)$.

We say the locally Lipschitz functional $h$ satisfies the nonsmooth Cerami condition if any sequence $\left\{x_{n}\right\}$ in $X$ such that $\left\{h\left(x_{n}\right)\right\}$ is bounded and $\left(1+\left\|x_{n}\right\|\right) \lambda\left(x_{n}\right) \rightarrow 0$ possesses a strongly convergent subsequence.

For convenience to quote we state some well known results, for more details, we can refer to $[3,8]$.

Lemma 2.1 ([5], Theorem 2.3.7). Let $x$ and $y$ be points in $X$, and suppose that $f$ is Lipschitz on open set containing the line segment $[x, y]$. Then there exists a point $u$ in $(x, y)$ such that

$$
f(y)-f(x) \in\langle\partial f(u), y-x\rangle .
$$

Lemma 2.2 ([6], Theorem 8). If $X$ is a reflexive Banach space, $X=Y \oplus V$ with $\operatorname{dim} Y<+\infty, \phi: X \rightarrow R$ is a locally Lipschitz function which is bounded from below, satisfies the nonsmooth Cerami condition, $\phi(0)=0$, $\inf _{X} \phi<0$ and there exists $r>0$ such that

$$
\begin{aligned}
& \phi(x) \leq 0, \quad \text { for } x \in Y,\|x\| \leq r, \\
& \phi(x) \geq 0, \quad \text { for } x \in V,\|x\| \leq r .
\end{aligned}
$$

Then $\phi$ has at least two nontrivial critical points.

\section{Proof of Theorems}

For every $u \in H_{T}^{1}$, let $\bar{u}=\frac{1}{T} \int_{0}^{T} u(t) d t, \tilde{u}(t)=u(t)-\bar{u}$. Then the following inequalities hold: 


$$
\begin{gathered}
\|\tilde{u}\|_{\infty}^{2} \leq \frac{T}{12} \int_{0}^{T}|\dot{u}(t)|^{2} d t, \quad \text { (Sobolev's inequality) } \\
\int_{0}^{T}|\tilde{u}(t)|^{2} d t \leq \frac{T^{2}}{4 \pi^{2}} \int_{0}^{T}|\dot{u}(t)|^{2} d t . \quad \text { (Wirtinger's inequality) }
\end{gathered}
$$

$$
\|u\|_{\infty} \leq C\|u\|
$$

where $C>0$ is a constant and $\|u\|_{\infty}=\max _{t \in[0, T]}|u(t)|$.

Define two functionals $\varphi_{1}, \varphi_{2}: H_{T}^{1} \rightarrow \mathbf{R}$ as follows:

$$
\varphi_{1}(u)=\frac{1}{2} \int_{0}^{T}|\dot{u}(t)|^{2} d t, \quad \varphi_{2}(u)=\int_{0}^{T} F(t, u(t)) d t .
$$

It is easy to verify that $\varphi_{1}$ is continuously differentiable and weakly lower semicontinuous (w.1.s.c.), and $\varphi_{2}$ is locally Lipschitz continuous on $H_{T}^{1}$. Since $H_{T}^{1}$ is embedded compactly and densely in $L^{2}\left(0, T ; \mathbf{R}^{N}\right)$, let $\hat{\varphi}_{2}: L^{2}\left(0, T ; \mathbf{R}^{N}\right) \rightarrow \mathbf{R}$ such that $\varphi_{2}=\left.\hat{\varphi}_{2}\right|_{H_{T}^{1}}$, then for every $u \in H_{T}^{1}, \xi \in \partial \varphi_{2}(u)$,

$$
\partial \varphi_{2}(u) \subseteq \partial \hat{\varphi}_{2}(u) \subseteq\left(L^{2}\left(0, T ; \mathbf{R}^{N}\right)\right)^{*}=L^{2}\left(0, T ; \mathbf{R}^{N}\right)
$$

and $\xi(t) \in \partial F(t, u(t))$ a.e. on $[0, T]$. Moreover,

$$
\left\langle\varphi_{1}^{\prime}(u), v\right\rangle=\int_{0}^{T}(\dot{u}(t), \dot{v}(t)) d t, \quad \forall u, v \in H_{T}^{1}
$$

Lemma 3.1. Let $F:[0, T] \times \mathbf{R}^{N} \rightarrow \mathbf{R}$ such that $F=F_{1}+F_{2}$, where $F_{1}, F_{2}$ satisfy assumption $\left(A^{\prime}\right),(3)$ and (4). Then the critical point of $\varphi$ corresponds to the solutions of problem (1).

Proof. From the condition (3), Obviously $F_{1}$ satisfies the Hypothesis $A$ of Theorem 2.7.5 in [5]. Since $f, g \in L^{\infty}\left(0, T ; \mathbf{R}^{+}\right)$, there exists a constant $c_{0}>0$ such that

$$
\eta \in \partial F_{2}(t, x) \Rightarrow|\eta| \leq f(t)|x|^{\alpha}+g(t) \leq c_{0}(|x|+1), \quad \forall x \in \mathbf{R}^{N}, t \in[0, T],
$$

i.e., $F_{2}$ satisfies the Hypothesis $B$ of Theorem 2.7.5 in [5]. Thus

$$
\partial\left(\int_{0}^{T} F_{1}(t, u) d t\right) \subset \int_{0}^{T} \partial F_{1}(t, u) d t, \quad \partial\left(\int_{0}^{T} F_{2}(t, u) d t\right) \subset \int_{0}^{T} \partial F_{2}(t, u) d t .
$$

Corollary 1 of Proposition 2.3.3 from [5] and (10) imply that, if at least one of the functions $F_{1}, F_{2}$ is strictly differentiable in $x$ for all $t \in[0, T]$ then for all $u \in H_{T}^{1}$,

$$
\begin{aligned}
\partial \varphi_{2}(u) & \subset \partial\left(\int_{0}^{T} F_{1}(t, u) d t\right)+\partial\left(\int_{0}^{T} F_{2}(t, u) d t\right) \\
& \subset \int_{0}^{T} \partial F_{1}(t, u) d t+\int_{0}^{T} \partial F_{2}(t, u) d t=\int_{0}^{T} \partial F(t, u) d t .
\end{aligned}
$$


Therefore, from (10) one has

$$
\begin{aligned}
\partial \varphi(u) & \subset \partial \varphi_{1}(u)+\partial \varphi_{2}(u) \\
& \subset \partial \varphi_{1}(u)+\int_{0}^{T} \partial F(t, u) d t \\
& =\partial \varphi_{1}(u)+\int_{0}^{T} \partial F_{1}(t, u) d t+\int_{0}^{T} \partial F_{2}(t, u) d t,
\end{aligned}
$$

i.e., for every $\xi \in \partial \varphi(u)$, there exist mappings $t \mapsto q(t)$ and $t \mapsto q_{i}(t) \quad(i=1,2)$ from $[0, T]$ to $\left(H_{T}^{1}\right)^{*}$ with $q(t) \in \partial F(t, u(t))$ and $q_{i}(t) \in \partial F_{i}(t, u(t))$ a.e. $t \in[0, T]$ such that for every $v \in H_{T}^{1}$,

$$
\begin{aligned}
\langle\xi, v\rangle & =\int_{0}^{T}(\dot{u}(t), \dot{v}(t)) d t+\int_{0}^{T}(q(t), v(t)) d t \\
& =\int_{0}^{T}(\dot{u}(t), \dot{v}(t)) d t+\int_{0}^{T}\left(q_{1}(t), v(t)\right) d t+\int_{0}^{T}\left(q_{2}(t), v(t)\right) d t .
\end{aligned}
$$

If $u \in H_{T}^{1}$ is a critical point of $\varphi$, then there exists $q_{0}(t) \in \partial F(t, u)$ such that for all $v \in H_{T}^{1}$,

$$
0=\langle\theta, v\rangle=\int_{0}^{T}(\dot{u}, \dot{v}) d t+\int_{0}^{T}\left(q_{0}(t), v(t)\right) d t .
$$

It follows easily that $q_{0}(t)=\ddot{u}(t)$ a.e. $t \in[0, T]$, thus

$$
\ddot{u}(t) \in \partial F(t, u(t)) \text { a.e. on }[0, T],
$$

which means that the critical point of $\varphi$ corresponds to the solutions of problem (1), which completes the proof.

Proof of Theorem 1.1. It follows from conditions (3), Hölder inequality and Wirtinger's inequality that

$$
\begin{aligned}
& \left|\int_{0}^{T} F_{1}(t, u(t)) d t-\int_{0}^{T} F_{1}(t, \bar{u}) d t\right| \\
& \quad \leq \int_{0}^{T}\left|F_{1}(t, u(t))-F_{1}(t, \bar{u})\right| d t \leq \int_{0}^{T} k(t)|\tilde{u}(t)| d t \\
& \quad \leq\left(\int_{0}^{T}|k(t)|^{2} d t\right)^{1 / 2}\left(\int_{0}^{T}|\tilde{u}(t)|^{2} d t\right)^{1 / 2} \\
& \quad \leq \frac{T}{2 \pi}\|k\|_{L^{2}}\left(\int_{0}^{T}|\dot{u}(t)|^{2} d t\right)^{1 / 2}
\end{aligned}
$$


for all $u \in H_{T}^{1}$. From Lemma 2.1 it follows that for each $t \in[0, T]$, there exist $s \in[0,1]$ and $\xi \in \partial F_{2}(t, \bar{u}+s \tilde{u})$ such that $F_{2}(t, u(t))-F_{2}(t, \bar{u})=(\xi(t), \tilde{u}(t))_{\mathbf{R}^{N}}$. By conditions (4) and Sobolev's inequality one has

$$
\begin{aligned}
& \left|\int_{0}^{T} F_{2}(t, u(t)) d t-\int_{0}^{T} F_{2}(t, \bar{u}) d t\right| \\
& \quad \leq \int_{0}^{T}\left|F_{2}(t, u(t))-F_{2}(t, \bar{u})\right| d t=\int_{0}^{T}|(\xi(t), \tilde{u}(t))| d t \\
& \quad \leq \int_{0}^{T}|\xi||\tilde{u}(t)| d t \leq \int_{0}^{T}\left(f(t)|\bar{u}+s \tilde{u}|^{\alpha}+g(t)\right)|\tilde{u}(t)| d t \\
& \quad \leq 2|\bar{u}|^{\alpha} \int_{0}^{T} f(t)|\tilde{u}(t)| d t+2\|\tilde{u}\|_{\infty}^{\alpha+1} \int_{0}^{T} f(t) d t+\|\tilde{u}\|_{\infty} \int_{0}^{T} g(t) d t \\
& \quad \leq \frac{3}{T}\|\tilde{u}\|_{\infty}^{2}+\frac{T}{3}|\bar{u}|^{2 \alpha}\left(\int_{0}^{T} f(t) d t\right)^{2}+2\|\tilde{u}\|_{\infty}^{\alpha+1} \int_{0}^{T} f(t) d t+\|\tilde{u}\|_{\infty} \int_{0}^{T} g(t) d t \\
& \quad \leq \frac{1}{4}\|\dot{u}\|_{L^{2}}^{2}+C_{1}\|\dot{u}\|_{L^{2}}^{\alpha+1}+C_{2}\|\dot{u}\|_{L^{2}}+C_{3}|\bar{u}|^{2 \alpha}
\end{aligned}
$$

for all $u \in H_{T}^{1}$ and some positive constants $C_{1}, C_{2}$ and $C_{3}$.

Hence from (5) we have

$$
\begin{aligned}
\varphi(u)= & \frac{1}{2} \int_{0}^{T}|\dot{u}(t)|^{2} d t+\left(\int_{0}^{T} F_{1}(t, u(t)) d t-\int_{0}^{T} F_{1}(t, \bar{u}) d t\right)+\int_{0}^{T} F_{1}(t, \bar{u}) d t \\
& +\left(\int_{0}^{T} F_{2}(t, u(t)) d t-\int_{0}^{T} F_{2}(t, \bar{u}) d t\right)+\int_{0}^{T} F_{2}(t, \bar{u}) d t \\
\geq & \frac{1}{2} \int_{0}^{T}|\dot{u}(t)|^{2} d t-\frac{T}{2 \pi}\|k\|_{L^{2}}\left(\int_{0}^{T}|\dot{u}(t)|^{2} d t\right)^{1 / 2}-\int_{0}^{T}|h(t)| d t-\frac{1}{4}\|\dot{u}\|_{L^{2}}^{2} \\
& -C_{1}\|\dot{u}\|_{L^{2}}^{\alpha+1}-C_{2}\|\dot{u}\|_{L^{2}}-C_{3}|\bar{u}|^{2 \alpha}+\int_{0}^{T} F_{2}(t, \bar{u}) d t \\
\geq & \frac{1}{4}\|\dot{u}\|_{L^{2}}^{2}-C_{1}\|\dot{u}\|_{L^{2}}^{\alpha+1}-\left(C_{2}+\frac{T}{2 \pi}\|k\|_{L^{2}}\right)\|\dot{u}\|_{L^{2}} \\
& +\int_{0}^{T} F_{2}(t, \bar{u}) d t-C_{3}|\bar{u}|^{2 \alpha}-C_{4} \\
= & \frac{1}{4}\|\dot{u}\|_{L^{2}}^{2}-C_{1}\|\dot{u}\|_{L^{2}}^{\alpha+1}-\left(C_{2}+\frac{T}{2 \pi}\|k\|_{L^{2}}\right)\|\dot{u}\|_{L^{2}}-C_{4} \\
& +|\bar{u}|^{2 \alpha}\left(|\bar{u}|^{-2 \alpha} \int_{0}^{T} F_{2}(t, \bar{u}) d t-C_{3}\right)
\end{aligned}
$$


for all $u \in H_{T}^{1}$ and some positive constant $C_{4}$, which implies that

$$
\varphi(u) \rightarrow+\infty \text { as }\|u\| \rightarrow \infty
$$

by (6) because $\alpha \in[0,1)$ and the norm $\|\cdot\|$ given by $\|u\|=\left(|\bar{u}|^{2}+\|\dot{u}\|_{L^{2}}^{2}\right)^{1 / 2}$ is an equivalent norm on $H_{T}^{1}$.

Next we show that the functional $\varphi$ is w.1.s.c. on $H_{T}^{1}$. Since $\varphi_{1}$ is w.1.s.c., we only have to prove that $\varphi_{2}$ is w.l.s.c. on $H_{T}^{1}$. Suppose there are a sequence $\left\{u_{n}\right\} \subseteq H_{T}^{1}$ and $u \in H_{T}^{1}$ such that $u_{n} \rightarrow u$ in $H_{T}^{1}$. Since the embedding $H_{T}^{1} \hookrightarrow$ $L^{2}\left([0, T], \mathbf{R}^{N}\right)$ is compact, one has $u_{n} \rightarrow u$ in $L^{2}\left([0, T], \mathbf{R}^{N}\right)$.

On account of (3), one has

$$
\begin{aligned}
& \left|\int_{0}^{T} F_{1}\left(t, u_{n}\right) d t-\int_{0}^{T} F_{1}(t, u) d t\right| \\
& \quad \leq \int_{0}^{T}\left|F_{1}\left(t, u_{n}\right)-F_{1}(t, u)\right| d t \leq \int_{0}^{T} k(t)\left|u_{n}-u\right| d t \\
& \quad \leq\left(\int_{0}^{T}|k(t)|^{2} d t\right)^{1 / 2}\left(\int_{0}^{T}\left|u_{n}-u\right|^{2} d t\right)^{1 / 2} \rightarrow 0
\end{aligned}
$$

Since $\alpha \in[0,1)$, there exists $C_{5} \in \mathbf{R}$ such that

$$
|x|^{2} \geq|x|^{2 \alpha}+C_{5}, \quad \forall x \in \mathbf{R}^{N} .
$$

Due to (4) and Lemma 2.1, there exist $\eta \in \partial F_{2}\left(t, s u_{n}+(1-s) u\right)$ and positive constants $C_{6}, C_{7}$ such that

$$
\begin{aligned}
\left|\int_{0}^{T} F_{2}\left(t, u_{n}\right) d t-\int_{0}^{T} F_{2}(t, u) d t\right| & \\
\leq & \int_{0}^{T}\left|F_{2}\left(t, u_{n}\right)-F_{2}(t, u)\right| d t=\int_{0}^{T}\left|\left(\eta(t), u_{n}(t)-u(t)\right)\right| d t \\
\leq & \int_{0}^{T}|\eta|\left|u_{n}-u\right| d t \leq \int_{0}^{T}\left(f(t)\left|s u_{n}+(1-s) u\right|^{\alpha}+g(t)\right)\left|u_{n}-u\right| d t \\
\leq & \int_{0}^{T}\left(2 f(t)\left(\left|u_{n}\right|^{\alpha}+|u|^{\alpha}\right)+g(t)\right)\left|u_{n}-u\right| d t \\
\leq & \left(2\|f\|_{\infty}\left(\left(\int_{0}^{T}\left|u_{n}\right|^{2 \alpha} d t\right)^{1 / 2}+\left(\int_{0}^{T}|u|^{2 \alpha} d t\right)^{1 / 2}\right)+\sqrt{T}\|g\|_{\infty}\right) \\
& \times\left(\int_{0}^{T}\left|u_{n}-u\right|^{2} d t\right)^{1 / 2} \\
\leq & \left(C_{6}\left(\left\|u_{n}\right\|_{L^{2}}+\|u\|_{L^{2}}\right)+C_{7}\right)\left\|u_{n}-u\right\|_{L^{2}} \rightarrow 0,
\end{aligned}
$$

which implies $\varphi_{2}\left(u_{n}\right) \rightarrow \varphi_{2}(u)$ in $H_{T}^{1}$. Thus $\varphi_{2}$ is sequentially weakly continuous; therefore, $\varphi$ is w.l.s.c. on $H_{T}^{1}$. Thanks to Theorem 1.1 and Corollary 1.1 in [7], $\varphi$ 
has a minimum $u_{0}$ on $H_{T}^{1}$. Proposition 2.3.2 in [5] implies that $u_{0}$ is a critical point of $\varphi$. Consequently, by Lemma 3.1, $u_{0}$ is a solution of problem (1), which completes the proof.

Proof of Theorem 1.2. Let us first note that $\varphi$ satisfies the nonsmooth Cerami condition. Pick a sequence $\left\{u_{n}\right\} \subset H_{T}^{1}$ such that $\left\{\varphi\left(u_{n}\right)\right\}$ is bounded and $\left(1+\left\|u_{n}\right\|\right) \lambda\left(u_{n}\right) \rightarrow 0$ as $n \rightarrow \infty$. By the weak* compactness of $\partial \varphi\left(u_{n}\right)$ and the weak lower semicontinuity of the norm, one can find $u_{n}^{*} \in \partial \varphi\left(u_{n}\right)$ such that $\lambda\left(u_{n}\right)=\left\|u_{n}^{*}\right\|=o(1)$, then there exists an integer $n_{0}$ such that for each $n \geq n_{0}$, we have

$$
\left|\left\langle u_{n}^{*}, v\right\rangle\right| \leq\|v\|, \quad \forall v \in H_{T}^{1} .
$$

Since $F_{1}, F_{2}$ satisfy the conditions of Theorem 2.7.5 in [5], one has $\partial \varphi_{2}(u) \subset$ $\int_{0}^{T} \partial F(t, u) d t$ and $\partial \varphi(u) \subset \partial \varphi_{1}(u)+\int_{0}^{T} \partial F(t, u) d t$. Thus to every $u_{n}^{*} \in \partial \varphi\left(u_{n}\right)$, there corresponds a mapping $t \mapsto q_{n}(t)$ from $[0, T]$ to $\left(H_{T}^{1}\right)^{*}$ with $q_{n}(t) \in$ $\partial F\left(t, u_{n}(t)\right)$ such that

$$
\left\langle u_{n}^{*}, v\right\rangle=\int_{0}^{T}\left(\dot{u}_{n}(t), \dot{v}(t)\right) d t+\int_{0}^{T}\left(q_{n}(t), v(t)\right) d t, \quad \forall v \in H_{T}^{1} .
$$

From the proof of Theorem 1.1 we know that $\varphi$ is coercive, which implies that the sequence $\left\{u_{n}\right\}$ turns out bounded. Thus there exists an $u \in H_{T}^{1}$ such that $u_{n} \rightarrow u$ in $H_{T}^{1}$ and $u_{n} \rightarrow u$ in $C\left([0, T], \mathbf{R}^{N}\right)$, where a subsequence is considered when necessary.

Since $H_{T}^{1}$ is reflexive while $\partial \varphi(u)$ is weak* compact, and the set-valued mapping $u \rightarrow \partial \varphi(u)$ is upper semicontinuous, we can find an $u^{*} \in \partial \varphi(u)$ such that

$$
\left\langle u_{n}^{*}-u^{*}, u_{n}-u\right\rangle \rightarrow 0, \quad \text { as } n \rightarrow \infty \text {. }
$$

Moreover

$$
\left\langle u_{n}^{*}-u^{*}, u_{n}-u\right\rangle=\int_{0}^{T}\left|\dot{u}_{n}(t)-\dot{u}\right|^{2} d t+\int_{0}^{T}\left(q_{n}(t)-q(t), u_{n}(t)-u(t)\right) d t,
$$

where $q_{n}(t) \in \partial F\left(t, u_{n}(t)\right)$ and $q(t) \in \partial F(t, u(t))$. Similarly, by the upper semicontinuity of the set-valued mapping $u \rightarrow \partial F(u)$, one has $q_{n}(t) \rightarrow q(t)$ in $w^{*} \quad$ topology and $\int_{0}^{T}\left(q_{n}(t)-q(t), u_{n}(t)-u(t)\right) d t \rightarrow 0$ as $n \rightarrow+\infty$. Thus $\int_{0}^{T}\left|\dot{u}_{n}-\dot{u}\right|^{2} d t \rightarrow 0$ and $u_{n} \rightarrow u$ in $H_{T}^{1}$. Therefore $\varphi$ satisfies the nonsmooth Cerami condition.

Now let $Y$ be a finite-dimensional subspace of $X=H_{T}^{1}$ given by

$$
Y=\left\{\sum_{j=0}^{k}\left(a_{j} \cos j \omega t+b_{j} \sin j \omega t\right) \mid a_{j}, b_{j} \in \mathbf{R}^{N}, j=0, \ldots, k\right\},
$$

and let $V=Y^{\perp}$. Then from (7) we have

$$
\varphi(u) \leq \frac{1}{2} \int_{0}^{T}|\dot{u}(t)|^{2} d t-\frac{1}{2} k^{2} \omega^{2} \int_{0}^{T}|u(t)|^{2} d t \leq 0,
$$


for all $u \in Y$ with $\|u\| \leq C^{-1} \delta$, and

$$
\varphi(u) \geq \frac{1}{2} \int_{0}^{T}|\dot{u}(t)|^{2} d t-\frac{1}{2}(k+1)^{2} \omega^{2} \int_{0}^{T}|u(t)|^{2} d t \geq 0
$$

for all $u \in V$ with $\|u\| \leq C^{-1} \delta$, where $C$ is the positive constant given by (11). Clearly $\varphi(0)=0$ because $\int_{0}^{T} F(t, 0) d t=0$, and $\varphi$ is bounded from below for it is coercive.

In the case $\inf _{X} \varphi<0$, Theorem 1.2 follows from Lemma 2.2.

In the case $\inf _{X} \varphi \geq 0$, according to (12) one has

$$
\varphi(u)=\inf _{X} \varphi=0
$$

for all $u \in Y$ with $\|u\| \leq C^{-1} \delta$, which implies that all $u \in Y$ with $\|u\| \leq C^{-1} \delta$ are minimum points of $\varphi$. Hence by Lemma 3.1, all $u \in Y$ with $\|u\| \leq C^{-1} \delta$ are solutions of problem (1). Therefore Theorem 1.2 is proved.

Proof of Theorem 1.3. Similar as in the proof of Theorem 1.2, we know $\varphi$ is bounded from below, satisfies the nonsmooth Cerami condition, and $\varphi(0)=0$.

According to the condition (8), we know for every $\varepsilon>0$ there exists $\delta_{1}>0$ such that

$$
F(t, x)>-\left(\frac{2 \pi^{2}}{T^{2}}+\varepsilon\right)|x|^{2}
$$

for a.e. $t \in[0, T]$ and $|x| \leq \delta_{1}$. Let $\delta_{2}=\min \left\{\delta_{1}, \hat{\delta}\right\}$, then from (9) one has

$$
-\left(\frac{2 \pi^{2}}{T^{2}}+\varepsilon\right)|x|^{2}<F(t, x) \quad \text { and } \quad \int_{0}^{T} F(t, x) d t \leq 0 \quad \forall|x| \leq \delta_{2}, t \in[0, T] .
$$

Let $H_{T}^{1}=\tilde{H}_{T}^{1} \oplus \mathbf{R}^{N}$ with $\tilde{H}_{T}^{1}=\left\{u \in H_{T}^{1} \mid \int_{0}^{T} u(t) d t=0\right\}$.

Since for every $u \in \tilde{H}_{T}^{1}$

$$
|u(t)|^{2} \leq\|u\|_{\infty}^{2} \leq \frac{T}{12} \int_{0}^{T}|\dot{u}(t)|^{2} d t \leq \frac{T}{12}\|u\|^{2} .
$$

Put $\delta_{3}=\min \left\{\sqrt{\frac{12}{T}} \delta_{2}, \hat{\delta}\right\}$, then for every $u \in \tilde{H}_{T}^{1}$ with $\|u\| \leq \delta_{3}$, one has $|u(t)| \leq \delta_{2}$ for all $t \in[0, T]$ and

$$
\begin{aligned}
\varphi(u) & =\frac{1}{2} \int_{0}^{T}|\dot{u}(t)|^{2} d t+\int_{0}^{T} F(t, u(t)) d t \\
& >\frac{1}{2} \int_{0}^{T}|\dot{u}(t)|^{2} d t-\left(\frac{2 \pi^{2}}{T^{2}}+\varepsilon\right) \int_{0}^{T}|u(t)|^{2} d t \\
& \geq \frac{1}{2} \int_{0}^{T}|\dot{u}(t)|^{2} d t-\left(\frac{2 \pi^{2}}{T^{2}}+\varepsilon\right) \frac{T^{2}}{4 \pi^{2}} \int_{0}^{T}|\dot{u}(t)|^{2} d t \\
& =-\frac{\varepsilon T^{2}}{4 \pi^{2}} \int_{0}^{T}|\dot{u}(t)|^{2} d t
\end{aligned}
$$


thus

$$
\begin{aligned}
\varphi(u) & \geq-\frac{\varepsilon T^{2}}{4 \pi^{2}} \int_{0}^{T}|\dot{u}(t)|^{2} d t \geq-\frac{\varepsilon T^{2}}{4 \pi^{2}}\|u\|^{2} \\
& \geq-\frac{\varepsilon T^{2}}{4 \pi^{2}} \delta_{3} \geq-\frac{\varepsilon T^{2}}{4 \pi^{2}} \hat{\delta},
\end{aligned}
$$

which implies that $\varphi(u) \geq 0$ for all $\|u\| \leq \delta_{3}$ in $\tilde{H}_{T}^{1}$ by the arbitrariness of $\varepsilon$.

On the other hand for every $u \in \mathbf{R}^{N}$ with $\|u\| \leq \delta_{3}$, it follows from (9) that

$$
\varphi(u)=\int_{0}^{T} F(t, u(t)) d t \leq 0 .
$$

Therefore, $\varphi$ satisfies the conditions of Lemma 2.2 and has at least two nontrivial critical points. With the critical point (global minima) obtained by Theorem 1.1 and taking Lemma 3.1 into account, problem (1) has at least three distinct solutions in $H_{T}^{1}$. The proof is completed.

Acknowledgement. The authors sincerely thank the referee for valuable suggestions and comments on the manuscript of this paper. The work was supported by the Fundamental Research Funds for the Central Universities (No: 2014B38214).

\section{REFERENCES}

[ 1 ] S. Aizicovici, N. S. Papageorgiou and V. Staicu, Multiple nontrivial solutions for nonlinear periodic problems with the $p$-Laplacian, J. Differential Equations 243 (2007), 504-535.

[2] N. Aizmahin And T. An, The existence of periodic solutions of non-autonomous second-order Hamiltonian systems, Nonliear Anal. 74 (2011), 4862-4867.

[ 3 ] G. Barletta AND S. A. Marano, Some remarks on critical point theory for locally Lipschitz functions, Glasgow Math. J. 45 (2003), 131-141.

[4] K. C. Chang, Variational methods for non-differentiable functionals and their applications to partial differential equations, J. Math. Anal. Appl. 80 (1981), 102-129.

[5] F. H. Clarke, Optimization and nonsmooth analysis, Classics Appl. Math. 5, SIAM, Philadephia, 1990.

[6] D. Kandilakis, N. Kourogenis and N. S. Papageorgiou, Two nontrivial critical points for nonsmooth functionals via local linking and applications, J. Global Optim. 34 (2006), 219-244.

[ 7 ] J. Mawhin And M. Willem, Critical point theory and Hamiltonian systems, Springer Verlag, New York, 1989.

[ 8 ] D. Motreanu and P. D. Panagiotopoulos, Minimax theorems and qualitative properties of the solutions of hemivariational inequalities, Nonconvex Optim. Appl. 29, Kluwer Acad., Dordrecht, 1998.

[ 9 ] E. H. Papageorgiou and N. S. Papageorgiou, Existence of solutions and of multiple solutions for nonlinear nonsmooth periodic systems, Czechoslovak Math. J. 54 (2004), 347-371.

[10] D. PAsca, Periodic solutions of a class of non-autonomous second-order differential inclusions systems, Abstr. Appl. Anal. 6 (2001), 151-161. 
[11] D. PASCA, Periodic solutions of second-order differential inclusions systems with $p$-Laplacian, J. Math. Anal. Appl. 325 (2007), 90-100.

[12] C. L. TANG, Periodic solutions for nonautonomous second systems with not sublinear nonlinearity, Proc. Amer. Math. Soc. 126 (1998), 3263-3270.

[13] C. L. Tang and X. P. Wu, Periodic solutions for second order Hamiltonian systems with not uniformly coercive potential, J. Math. Anal. Appl. 259 (2001), 386-397.

Yan Ning

COLlege of SCIENCE

HOHAI UNIVERSITY

NANJING 210098

P.R. CHINA

E-mail: ningkegood@126.com

Tianqing An

College of Science

HoHai UNIVERSITY

NANJING 210098

P.R. CHINA

E-mail: antq@hhu.edu.cn 